\title{
Electrones inteligentes
}

\author{
José Roberto Víctor Manuel Salas Barboza \\ ingjrvictor@gmail.com \\ Investigador Independiente \\ Puno - Perú
}

\section{RESUMEN}

Cuando hay una diferencia de presión en algún lugar de la atmósfera, empieza la lluvia entonces las gotas que caen, ruedan por las laderas y quebradas juntándose en lugares aparentes para formar lagos de donde salen los ríos, que llegan a los océanos. Allí se evaporan y se condensan construyendo las nubes que el viento aleja hacia las montañas donde repiten la secuencia. Por otro lado, las semillas en la tierra originan plantas o árboles que florecen y que, según su especie, producen frutos de los cuales caen los granos que reinciden la secuencia. Ambas rutinas nos pasan desapercibidas porque son parte de la naturaleza y estamos acostumbrados a ellas. Los electrones que llegan del Sol quedan atrapados por el campo magnético y giran entre los polos formando toroides electromagnéticos generando otro ciclo que permite que se desarrolle la vida tal como fue concebida en los planetas que tienen condiciones como la Tierra, nuestro mundo.

Palabras clave: teoría; luz; gravedad; atracción magnética 


\title{
Smart electrons
}

\begin{abstract}
When there is a pressure difference somewhere in the atmosphere, the rain begins so the drops that fall, roll down the slopes and ravines, joining together in apparent places to form lakes from where the rivers flow, reaching the oceans. There they evaporate and condense, building the clouds that the wind pushes towards the mountains where they repeat the sequence. On the other hand, the seeds in the ground originate plants or trees that bloom and that, according to their species, produce fruits from which the grains that recur the sequence fall. Both routines do not go unnoticed because they are part of nature and we are used to them. The electrons that come from the Sun are trapped by the magnetic field and rotate between the poles forming electromagnetic toroids, generating another cycle that allows life to develop as it was conceived on planets that have conditions like Earth, our world.
\end{abstract}

Keywords: theory; light; gravity; magnetic attraction

Artículo recibido: 30 noviembre. 2021 Aceptado para publicación: 29 diciembre 2021

Correspondencia: ingjrvictor@gmail.com Conflictos de Interés: Ninguna que declarar 


\section{INTRODUCCIÓN}

En 1785, C.A. Coulomb descubrió la conductividad del aire. Su descubrimiento fue contrario a la idea prevaleciente en ese momento de que los gases atmosféricos eran aislantes (que son, en cierta medida, o al menos no muy buenos conductores cuando no están ionizados). Desafortunadamente, su investigación fue completamente ignorada. P. Erman (1804) teorizó que la Tierra estaba cargada negativamente. Peltier (1842) probó y confirmó la idea de su predecesor. Los descubrimientos sobre la electrificación de la atmósfera a través de instrumentos eléctricos sensibles e ideas sobre cómo se mantiene la carga negativa de la Tierra se han desarrollado principalmente en el siglo XX. Si bien se ha realizado una cierta cantidad de trabajo de observación en las ramas de la electricidad atmosférica, la ciencia no se ha desarrollado en gran medida. La prueba COEL muestra que la Tierra tiene el signo positivo (+).

La naturaleza física de la luz ha sido uno de los grandes problemas de la ciencia. Desde la antigua Grecia, la luz se consideraba algo de naturaleza corpuscular, eran pizcas que formaban el rayo de luz. Así explicaron fenómenos como la reflexión y la refracción de la luz. Isaac Newton en el siglo XVIII defendió esta idea, asumió que la luz estaba formada por aquellas arrojados a gran velocidad por los cuerpos emisores de luz. Escribió un tratado de óptica en el que explicaba una multitud de fenómenos sufridos por la luz.

En 1678 Christiaan Huygens defiende un modelo de onda y la luz era una. Con este modelo, se explicaron fenómenos como la interferencia y la difracción que el modelo corpuscular no pudo explicar. Así, la luz era una onda longitudinal, pero las ondas longitudinales necesitan un medio para propagarse, y el concepto de éter surgió como el "medio" en el que estamos inmersos. Esto trajo aún más problemas, y la naturaleza del éter fue un dolor de cabeza para muchos científicos.

La solución fue dada por James Clerk Maxwell en 1865, la luz es una onda electromagnética que se propaga en el vacío. Por lo tanto, se resolvió el problema del éter con la aparición de estas nuevas ondas. Quién se basó en los estudios de Faraday sobre electromagnetismo y concluyó que las ondas de luz son de naturaleza electromagnética. Una ONDA ELECTROMAGNÉTICA es producida por la variación en algún lugar del espacio de las propiedades eléctricas y magnéticas de la materia. No necesita ningún medio para extenderse, son ondas transversales. 
Una carga eléctrica que oscila con cierta frecuencia produce ondas electromagnéticas de la misma frecuencia. La velocidad con la que estas ondas se propagan en el vacío es: $\mathrm{c}=$ $3 \times 10^{8} \mathrm{~m} / \mathrm{s}$, que es un límite que aun no sido igualado por nuestra tecnología.

Las diferentes teorías que definen la luz de una forma u otra manipulan electrones que producen reflexión, refracción, difracción, ondas magnéticas, ondas electromagnéticas o electricidad, como veremos más adelante.

\section{Magnetismo}

Aunque la electricidad y el magnetismo son dos ramas completamente diferentes, el hecho de poder manipular electrones, que es el enlace común, los acerca más y quizás algo sorprendente suceda más tarde.

El fenómeno del magnetismo fue conocido por los antiguos griegos durante más de 2000 años. Se observó que ciertos minerales (imanes) podrían atraer o repeler pequeños objetos de hierro. De hecho, el nombre del magnetismo viene de la provincia griega de Magnesia, donde se encuentran los depósitos más importantes de magnetita (Fe3O4), mineral con fuertes propiedades magnéticas.

Aunque este fenómeno se conoció experimentalmente, no fue hasta mediados del siglo XIX que todas las interacciones de tipo eléctrico y magnético, resumidas en las ecuaciones de Maxwell, se formularon teóricamente.

Las propiedades magnéticas son más pronunciadas en los extremos del imán, que se llaman polos magnéticos, Polo Norte (N) y Sur (S). De la misma manera que las cargas eléctricas del mismo signo se repelen y atraen de manera diferente, los imanes que se acercan por polos iguales se repelen y si se acercan por polos opuestos, se atraen. Es imposible aislar un solo polo magnético, de modo que si un imán se divide en dos, en cada pieza nuevamente hay un polo norte y un polo sur.

Cuando se trabaja con tubos de ensayo, a veces hay una burbuja de aire que no desaparece e intriga a los investigadores que hicieron teorías sobre por qué sucedió eso. Un estudiante resolvió el enigma que comenzó a observar, no sabemos cuántos días, pero se dio cuenta de que estaba comenzando a ascender tan lentamente que era imperceptible y después de un tiempo llega a la superficie desapareciéndo y revela que no hay mejor manera de resolver las hipótesis que con los experimentos. Y afirma que investigar requiere tiempo, paciencia, conocimiento, dominio del tema e inspiración. 


\section{El Sol}

Es la estrella más cercana a la Tierra, y es el centro de nuestro Sistema Solar, una bola gigante giratoria de gas muy caliente, es energizada por reacciones de fusión nuclear, calienta nuestro planeta y hace posible la vida. También es una estrella activa con rayos y manchas solares, prominencias y eyecciones de masa coronal. Estos fenómenos, todos relacionados con el campo magnético, impactan el espacio cerca de la Tierra y determinan nuestro "clima espacial". En unos cinco mil millones de años, se convertirá en un gigante rojo $\mathrm{y}$, finalmente, en una estrella enana blanca. Muchas culturas han tenido mitos interesantes sobre él, reconociendo su importancia para la vida en la Tierra. No podemos ver el interior, por lo que los científicos usan otros diagnósticos que nos ayudan a saber qué hay dentro. Para entender cómo funciona, ayuda imaginar el interior formado por diferentes capas, una dentro de la otra. El núcleo, o centro, es la región donde se produce energía. En la Tierra sabemos que la produce porque vemos la luz y sentimos calor en un día de verano. Y a medida que se produce en el núcleo, viaja hacia afuera y primero a través de la zona radiactiva, donde las partículas de luz (fotones) transportan energía. Un fotón tarda millones de años en alcanzar la siguiente capa, la zona de convección, en esta, la energía viaja más rápido. Ahora son los movimientos de los gases del Sol los que transportan la energía hacia afuera. El gas en esta capa se mezcla y burbujea, como el movimiento de una olla de agua hirviendo. Este efecto burbujeante se ve en su superficie y se llama granulación.

El Sol (del latín sol, solis, "dios Sol invictus" o "sol", Helios en la mitología griega, a su vez de la raíz proto-indoeuropea sauel-, "brillo") es una estrella de tipo G de La secuencia principal y la clase de luminosidad V que se encuentra en el centro del sistema solar y constituye la mayor fuente de radiación electromagnética de este sistema planetario. Es una esfera de plasma casi perfecta, con un movimiento convectivo interno que genera un campo magnético a través de Un proceso de dinamo. Alrededor de tres cuartos de su masa consiste en hidrógeno; el resto es principalmente helio, con cantidades mucho más pequeñas de elementos, incluidos oxígeno, carbono, neón y hierro.

Se formó hace aproximadamente 4600 millones de años a partir del colapso gravitacional de la materia dentro de una región de una gran nube molecular. La mayor parte de esta materia se acumuló en el centro, mientras que el resto se aplanó en un disco en órbita que se convirtió en el sistema solar. La masa central se volvió cada vez más densa y caliente, 
lo que eventualmente condujo al comienzo de la fusión nuclear en su núcleo. Se cree que casi todas las estrellas están formadas por este proceso. Se trata de la mediana edad y no ha cambiado drásticamente durante más de cuatro mil millones de años, y se mantendrá bastante estable durante otros cinco mil millones de años. Sin embargo, después de que la fusión del hidrógeno en su núcleo se haya detenido, sufrirá grandes cambios y se convertirá en un gigante rojo. Se estima que será lo suficientemente grande como para engullir las órbitas actuales de Mercurio, Venus y posiblemente la Tierra.

Los planetas y otros cuerpos (incluidos, asteroides, meteoroides, cometas y polvo) orbitan a su alrededor. En sí mismo, representa aproximadamente el $99.86 \%$ de la masa del sistema solar. La distancia promedio a la Tierra fue definida exactamente por la Unión Astronómica Internacional en 149, 597, 870,700 metros (aproximadamente 150 millones de kilómetros). Su luz recorre esta distancia en 8 minutos y 20 segundos.

Su energía en forma de luz solar, soporta casi todas las formas de vida a través de la fotosíntesis, y determina el clima y la meteorología del globo terraqueo. Es la estrella principal del sistema planetario en que nos encontramos; por lo tanto, posee el mayor brillo aparente. Su visibilidad en el cielo local determina, respectivamente, día y noche en diferentes regiones de diferentes planetas. En la Tierra, la energía irradiada por el Sol es aprovechada por los seres fotosintéticos que constituyen la base de la cadena alimentaria, siendo así la principal fuente de energía de la vida. También proporciona la esnergía que mantiene en funcionamiento los procesos climáticos.

Se encuentra en la fase llamada secuencia principal, con un tipo espectral G2 y una clase de luminosidad V, por lo tanto, también se llama enana amarilla, formada entre 4567.9 y 4570.1 millones de años y permanecerá en la secuencia principal aproximadamente 5000 millones de años más. Y junto con todos los cuerpos celestes que orbitan a su alrededor, incluida la Tierra, forman el sistema solar. Y es el único cuya forma se puede ver a simple vista, con un diámetro angular de 32'35 " de arco en el perihelio y 31'31" en el afelio, lo que da un diámetro promedio de 32'03". La combinación de tamaños y distancias del Sol y la Luna son tales que se ven, aproximadamente, con el mismo tamaño aparente en el cielo. Esto permite una amplia gama de eclipses solares diferentes (total, anular o parcial). El vasto efecto en la Tierra ha sido reconocido desde tiempos prehistóricos y algunas culturas lo han considerado como una deidad. El movimiento de la Tierra a su alrededor es la base del calendario solar, que se usa predominantemente en la actualidad. 
La disciplina científica responsable de su estudio en su totalidad es la física solar.

Características.- Es una estrella de tipo $G$ de la secuencia principal que cubre aproximadamente el $99.86 \%$ de la masa del sistema solar. Y tiene una magnitud absoluta de +4.83 , estimada como más brillante que el $85 \%$ de las estrellas de la Vía Láctea, la mayoría de las cuales son enanas rojas. Pertenece a la Población I, o a las estrellas ricas en elementos pesados. Su formación puede haber sido causada por ondas de choque de una o más supernovas cercanas. Esto se planteó debido a la gran abundancia de elementos pesados en el sistema solar, como el oro y el uranio, en relación con la abundancia de estos elementos en la llamada Población II de estrellas, que son pobres en elementos pesados. Estos elementos podrían haber sido producidos por reacciones nucleares endotérmicas durante una supernova, o por transmutación a través de la absorción de neutrones dentro de una estrella masiva de segunda generación. Y es, con diferencia, el objeto más brillante del cielo, con una magnitud aparente de -26,74. Es aproximadamente 13 mil millones de veces más brillante que la segunda estrella más brillante, Sirio, que tiene una magnitud aparente de -1.46. La distancia promedio desde su centro al centro de la Tierra es de aproximadamente 1 unidad astronómica (aproximadamente 150 millones de kilómetros), aunque la distancia varía a medida que la Tierra se mueve desde el perihelio en enero hasta el afelio en julio. En esta distancia promedio, la luz viaja desde su horizonte hasta el de la Tierra en aproximadamente 8 minutos y 19 segundos, mientras que la luz desde los puntos más cercanos del Sol y la Tierra tarda aproximadamente dos segundos menos.

No tiene un límite definido y en sus partes externas su densidad disminuye exponencialmente a medida que aumenta la distancia desde su centro. Sin embargo, para fines de medición, el radio solar se considera como la distancia entre su centro y el borde de la fotósfera, la superficie visible visible de la estrella. Según esta medida, es una esfera casi perfecta con un aplanamiento estimado de 9 millonésimas, lo que significa que su diámetro polar difiere de su diámetro ecuatorial en solo 10 kilómetros. El efecto de marea de los planetas es débil y no afecta significativamente su forma y gira más rápido por su ecuador que por sus polos. Esta rotación diferencial es causada por el movimiento de convección debido al transporte de calor y el efecto coriolis producido por su rotación. En un marco definido por las estrellas, el período de rotación es de aproximadamente 25,6 
días en el ecuador y 33,5 días en los polos. Visto desde la Tierra en su órbita, el período de rotación aparente en su ecuador es de aproximadamente 28 días.

Luz solar.- La constante solar es la cantidad de energía depositada por unidad de tiempo y superficie y que está directamente expuesta a la luz solar. La constante solar es igual a aproximadamente $1368 \mathrm{~W} / \mathrm{m}^{2}$ (vatios por metro cuadrado) a una distancia de una unidad astronómica (ua) del Sol (es decir, en la Tierra o a la misma distancia del Sol como está). La luz que llega a la Tierra es manipulada por la atmósfera de tal modo que genera electrones libres y electricidad (aproximadamente $1000 \mathrm{~W} / \mathrm{m}^{2}$ ) en condiciones claras cuando está cerca del cenit que permite la vida orgánica. En la parte superior está compuesta (por energía total) de aproximadamente 50\% de infrarroja, $40 \%$ de visible y $10 \%$ de ultravioleta. Y filtra más del 70\% de la radiación solar ultravioleta, especialmente a longitudes de onda más cortas. La radiación ultravioleta solar ioniza la parte superior de la atmósfera diurna, lo que hace que la ionosfera sea conductiva para electricidad.

$\mathrm{Su}$ color es blanco con un índice de color al espacio (ICD) cercano a $(0.3 ; 0.3)$ cuando se ve desde el espacio o desde lo alto del cielo; sin embargo, cuando es de un área baja del cielo, su dispersión atmosférica tiene un color amarillo, rojo, naranja y magenta. A pesar de su blancura típica, la mayoría de la gente lo imagina como amarillo; Las razones de esto son debatidas. Es una estrella G2V, con G2 que indica que su temperatura superficial es de aproximadamente $5778 \mathrm{~K}\left(5505^{\circ} \mathrm{C}\right)$ y V que, como la mayoría de las estrellas, es una estrella enana de la secuencia principal. La luminosidad promedio del Sol es de aproximadamente $1.88 \mathrm{Gcd} / \mathrm{m}^{2}$, pero como se ve a través de la atmósfera de la Tierra, esto se reduce a aproximadamente $1.44 \mathrm{Gcd} / \mathrm{m}^{2}$. Sin embargo, la luminancia no es constante a través del disco del Sol (oscurecimiento del limbo).

Composición. - Se compone principalmente de los elementos químicos hidrógeno y helio; que representan el $74.9 \%$ y el $23.8 \%$ de la masa del Sol en la fotósfera, respectivamente. Todos los elementos más pesados, llamados metales en astronomía, representan menos del $2 \%$ de la masa, con oxígeno (más o menos $1 \%$ de su masa), carbono $(0.3 \%)$, neón $(0.2 \%)$ y hierro $(0.2 \%)$ siendo el más abundante

Heredó su composición química del medio interestelar a través del cual se formó. El hidrógeno y el helio se produjeron por nucleosíntesis y los elementos más pesados se crearon por la misma razón en generaciones de estrellas que completaron su evolución estelar y devolvieron su material al medio interestelar antes de su formación. La 
composición química de la fotosfera normalmente se considera representativa de la composición del sistema solar primario. Sin embargo, desde que se formó, parte del helio y elementos pesados se han asentado gravitacionalmente desde la fotósfera. Por lo tanto, en la actual, la fracción de helio se reduce y la capacidad es solo el $84 \%$ de lo que era en la fase protoestelar (antes de que comenzara la fusión nuclear en el núcleo). Se cree que la composición del Sol ha sido $71.1 \%$ de hidrógeno, $27.4 \%$ de helio y $1.5 \%$ de elementos más pesados.

Hoy en día, la fusión nuclear en su núcleo ha modificado la composición al convertir hidrógeno en helio, por lo que ahora la parte más interna es aproximadamente $60 \%$ de helio, junto con la abundancia de elementos más pesados sin ser alterados porque el calor se transfiere desde el centro por radiación en lugar de convección., ninguno de los principales productos de fusión ha llegado a la fotosfera.

La zona reactiva del núcleo de "combustión de hidrógeno", donde el hidrógeno se convierte en helio, está comenzando a estar rodeada por un núcleo interno de "cenizas de helio". Este desarrollo continuará y luego su salida de la secuencia principal tendrá lugar para convertirse en un gigante rojo.

La abundancia de elementos pesados solares descritos anteriormente se mide utilizando la espectroscopía de su fotósfera y midiendo las abundancias en meteoritos que nunca se han calentado a temperaturas de fusión. Se cree que estos meteoritos conservan su composición protoestelar y, por lo tanto, no se ven afectados por la sedimentación de elementos pesados. Por lo general, los dos métodos coinciden bien.

\section{Parte Experimental}

Desde la secundaria era una intriga cómo en los vegetales la savia asciende a través del xilema, las explicaciones en ciencias naturales funcionan para elevaciones de menos de 10 metros, los millones de árboles que existen en los bosques del planeta y alcanzan los 120 no lo tenían porque no eran universales; para serlo, deberían trabajar en cualquier altura. Aunque nunca resolvió la duda y no había necesidad de saberlo porque las cosas y la vida funcionan sin ella, todo el mundo inclusive los investigadores las tomaron como verdad.

Con el tiempo y el trabajo de ingeniería olvidó el asunto, hubo problemas que requirieron su atención. Y fue así que años después, cuando viajaba en automóvil, sufrió un accidente, estaba inconscientemente y conectado a una máquina, sin daños tanto en la columna 
vertebral como el cerebro, era cuestión de tiempo que abra los ojos, lo que sucedió en un hospital después de ocho días. El accidente tuvo secuelas, no sentía el cuerpo tuvo amnesia temporal y al mirar los recuerdos, vino a su mente esa pregunta de la escuela secundaria y decidió investigarlo.

Preparó la prueba COEL (por sus siglas en español Colector de Electrones) donde imitando lo que sucede en la naturaleza con las hojas de los vegetales. Hizo una placa de plata de 3 x 4 × $0.1 \mathrm{~cm} 3$ conectada por cable a un LED de $3 \mathrm{~W}$ y cuando se enciende los electrones lo atraviesan e iluminan. Como lo capturado excedía, el porcentaje teórico, de acuerdo con lo que sucedió tuvo que redefinir el concepto de electricidad. Las diferentes teorías que explican la luz no permiten comprender que la naturaleza los maneja como flujo de electrones que generan luz, calor, magnetismo, ondas electromagnéticas y este ensayo los captura por su polaridad (-) demostrando que la Tierra tiene el signo opuesto.

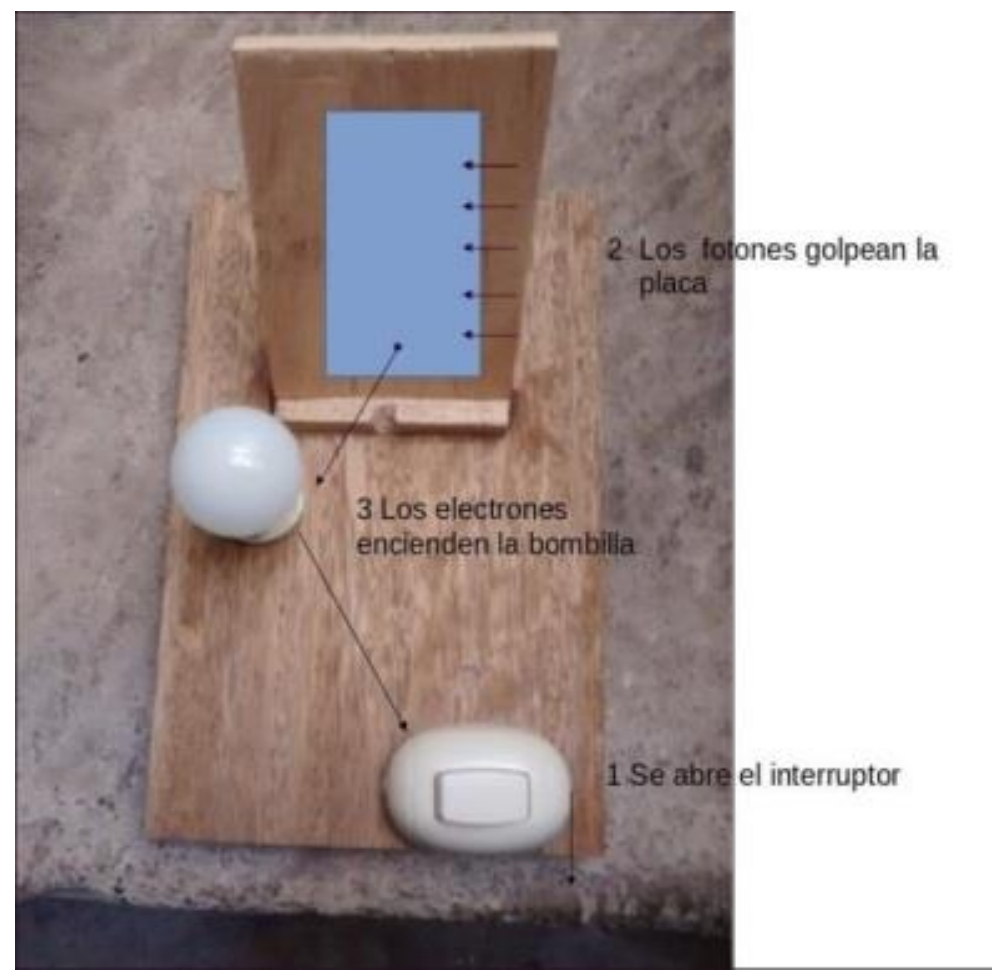

\section{RESULTADOS Y DISCUSIONES}

1. El flujo de electrones que manipula la naturaleza y origina reflexión, refracción, difracción, luz, gravedad y llega al polo opuesto se llama electricidad

2. La temperatura elevada del Sol fusiona electrones que pierden masa pero ganan energía y se alejan como fotones cuando atraviesan la atmósfera, son manipulados 
por la naturaleza, liberando calor, color y electricidad que ingresa a los vegetales a producir oxígeno, dar vida a los animales y conciencia al ser humano.

3. Los fotones no tienen masa pero manipulan la polaridad y se alejan porque se repelen. Esto explica el corrimiento al rojo que Edwin Hubble observó, transportan carga negativa. Esta prueba nos demuestra y enseña que es posible manipularlos como lo hace la naturaleza.

4. En planetas con condiciones similares al nuestro podemos sembar la vida que el Creador puso en la Tierra.

5. Toda materia se oxida perdiendo electrones que son atrapados por los planetas. Y por ello nos demuestran que tienen signo opuesto.

6. Un metro cúbico de aire pesa más que su similar de agua. Por sentido común debería estar abajo pero como es gas y mas ligero figura encima envolviendo al planeta e impidiendo que aquella escape al espacio por la atracción magnética que ejerce la Tierra sobre todos los cuerpos y conocemos como gravedad.

\section{CONCLUSIONES}

I. El flujo de electrones que giran indefinidamente en la Tierra genera energía y esta la vida tal cual conocemos

II. La gravededad es atracción mag magnética. Cuando finaliza un eclipse los rayos que rozan la atmosfera se curvan y alejan siguiendo el perfil del globo terrestre por la atraccion-repulsion magnetica que ejerce la Tierra

III. La elevada temperatura fusiona electrones que se alejan del Sol por su tiempo polaridad como fotones, que no tienen masa que aparece cuando la manipulan, ello explica el corrimiento al rojo que observó Edwin Hubble. Cuando atraviesan la atmósfera, liberan calor, color y electricidad que ingresa a los vegetales para producir oxígeno, vivan los animales y se desarrolle el ser humano..

IV. La gravedad es atracción magnética y aplicando las leyes de la termodinámica podemos generar vida en planetas con condiciones similares..

V. Experimentalmente, Hans Christian Oersted (1777-1815) descubrió la existencia de un campo magnético alrededor de cada conductor de electricidad. Los rayos del Sol que no caen verticalmente, son refractados por el ángulo, pero atraídos por el signo y así sucesivamente, siguen el perfil del globo hasta que se alejan del campo magnético 
y retoman su trayectoria inicial. La curva que describen al rozar la Tierra revela que no es plana y los fotones transportan electricidad.

VI. Si el Universo tuvo un principio retrocediendo por el camino dejado entre las estrellas, llegaríamos a èl. Como se expande en todas las direcciones y no lo hay, eso significa que es infinito, un concepto difícil de entender al incrédulo pues da la impresión que la materia siempre existió. Lo cual es falso. Hay un Creador, la prueba de esto es el ser humano porque lo hizo a su imagen y semejanza.

VII. En el mundo cuántico, los eventos se repiten en niveles más profundos de la materia si asumimos que en el Universo sucede lo mismo implicaría que hay más Sistemas Solares y que, conociendo estos principios, los habitaremos para que seamos como las estrellas.

\section{LISTA DE REFERENCIAS}

Newton Isaac, “Opticks: or, a treatise of the reflections, refractions”, London, Sam Smith and Benj Walford, Reino Unido, 1704..

Huygens Christiaan, “Treatise on Light, London”, CreateSpace Independent Publishing Platform, Londres, Reino Unido, 1690.

Maxwell, James Clerk, "A treatise on electricity and magnetism”, London, US, LIGHTNING SOURCE, Londres, Reino Unido, 1873.

Stewart Gillmor, Coulomb, "The Evolution of Physics and Engineering in EighteenthCentury" France, Princeton, Princeton University Press, 1971.

VALENZUELA, I. M. E. M., MAY, D. A. L. H., \& SANTILLÁN, D. A. D. L. DiSEÑO DE GENERADORES HÍBRIDOS BASADOS EN ENERGÍA SOLAR Y AGUA DE LLUVIA PARA EDIFICIOS INTELIGENTES.

FLORES, J. J. M. (2012). Aleaciones Ferromagnéticas Para Aplicación como Barreras Térmicas Inteligentes.

Galván, V., Limandri, S., Gargicevich, D., Bonifacich, F. G., Lambri, O. A., Blanco, C., ... \& Cuello, G. J. ESTUDIO DE LAS FASES EN Fe-Al-Ge MEDIANTE DIFRACCION DE ELECTRONES RETRODISPERSADOS Y NEUTRONES.

Jara Uribe, D. A. (2020). Preparación y caracterización de hidrogeles inteligentes responsivos a campos magnéticos.

Vázquez Acosta, E. N. (2008). Diseño de etapa de amplificación y adecuación de señal en un circuito integrado para un micro sensor de gases (MEMS) inteligente. 
TOVAR, G. N., HAidÉ, J., CASTILlo, M., \& CASTILlO, M. D. C. G. La ropa inteligente.

VARGAS, J., GIRALDO, F., \& ISAAC, I. (2015). Implementación de un Generador Solar Fotovoltaico con Microinversores en la Micro-Red Inteligente de la Universidad Pontificia Bolivariana (UPB). Ingeniar UPB. Medellín, 4, 3-16.

Otero, T. F. (1997). Los polímeros conductores como materiales biomiméticos. Bol Soc Esp Cerám y Vidrio, 36, 230-237.

Ortiz Dosal, L. C., \& Ángeles Robles, G. (2018). ¿ Qué son los materiales inteligentes?. Madina-Doñabeitia, C., \& Arechalde-Ugarteche, I. (2011). Las nuevas redes eléctricas inteligentes, su medida y gestión de la demanda. DYNA-Ingeniería e Industria, 86(3).

Amashta, I. A. K. Hidrogeles inteligentes.

Menéndez, J. E. Á. (2013). Iluminación led en edificios inteligentes. Libro de comunicaciones, 202.

GLADYS, D. L. S. V., HERRERA, L. E. E., \& BELMONTES, F. A. (2012). Compuestos fotocromáticos: moléculas inteligentes y con buena memoria.

Islas Silvas, O. A. (2019). Algoritmo inteligente para la optimización del proceso de carga en una batería LiPo.

Fernández Otero, T. (1995). Nuevas soluciones para viejos problemas: baterías poliméricas, ventanas inteligentes y músculos artificiales.

Goilav, N., \& Geoffrey, L. O. I. (2016). Arduino: Aprender a desarrollar para crear objetos inteligentes. Ediciones ENI.

\section{Links de interes}

https://youtu.be/m0FC13eLi5M

https://vimeo.com/377523344 